\title{
Contribuciones del paradigma cultural latinoamericano a la comunicación para el desarrollo. Antecedentes, textos y contextos de una relación fecunda*
}

\author{
Rafael Ángel Salazar Martínez \\ Rayza Portal Moreno*** \\ Rafael Ángel Fonseca Valido ${ }^{* \star * *}$
}

Recibido: 2016-03-20. Enviado a pares: 2016-05-09 Aprobado por pares: 2016-05-18. Aceptado: 2016-06-09

DOI: 10.22395/angr.v15n29a1

\begin{abstract}
Resumen
Durante la década de 1980 se produce una ruptura en la investigación comunicológica latinoamericana, cuyo resultado fue el nacimiento de un nuevo paradigma que se apropia y hace uso de una concepción antropológica de la cultura, desde la cual comienzan a abordarse los procesos comunicativos. Las contribuciones de este paradigma a la comunicación para el desarrollo, campo que en América Latina ya contaba con una larga tradición, han resultado particularmente significativas. A examinar y discutir sus principales antecedentes, así como a identificar y esclarecer las relaciones entre ellas, dedicamos las páginas del trabajo.

Palabras clave: Paradigma cultural latinoamericano, contribuciones, comunicación para el desarrollo, campo, ruptura.
\end{abstract}

\footnotetext{
El trabajo es un artículo de revisión resultado del marco teórico de la tesis y proyecto de investigación doctoral Programas Integrales de desarrollo municipal: coordenadas para una articulación de sus procesos comunicativos financiado por la Universidad de Holguín "Oscar Lucero Mora" y desarrollado por el autor principal desde la Facultad de Comunicación de la Universidad de La Habana, con la tutoría de ambos coautores (CUBA).

** $\quad$ Profesor asistente e investigador del Centro Universitario Municipal de Mayarí, adscrito a la Universidad de Holguín "Oscar Lucero Moya", Cuba. Maestro en Educación Superior, y doctorando en Ciencias de la Comunicación Social por la Universidad de La Habana. Correo electrónico: rasalazarm@cum.uho.edu.cu.

*** Profesora titular y jefa de la Disciplina de Comunicación y Desarrollo en la Facultad de Comunicación de la Universidad de La Habana. Doctora en Ciencias de la Comunicación por el mismo centro formador Correo electrónico: rayza@fcom.uh.cu.

*** Profesor titular del Departamento de Comunicación Social de la Universidad de Oriente, Cuba. Doctor en Ciencias de la Comunicación por la Universidad de La Habana. Correo electrónico: rfvalido@enet.cu
} 


\title{
Latin-American Cultural Paradigm Contributions to Communication for the Development, Background, Texts, and Contexts of a Productive Relation
}

\begin{abstract}
During 1980 decade, there was a rupture in Latin-American communication research, which result was the birth of a new paradigm, which uses an anthropologic conception of culture, from which communicative processes start to be tackled. Contributions of this paradigm to communication for the development, a field that already had a long tradition in Latin America Latina, have been particularly meaningful. This work is devoted to examine and discuss their main backgrounds and to identify and clarify relations among them.
\end{abstract}

Key words: Latin American cultural paradigm, contributions, communication for the development, field, rupture. 


\section{Introducción}

No son pocos los trabajos que han intentado dar cuenta, en todo o en parte, con mayor o menor acierto, del camino recorrido por la comunicación para el desarrollo en su más de medio siglo de fecunda existencia. Sin pretender llover nuevamente sobre un terreno ya de por sí algo saturado, consideramos necesario realizar, luego de las coordenadas metodológicas, nuestro propio acercamiento a los antecedentes de la temática central del artículo, cuyo examen y discusión permitirá obtener una visión más acabada sobre la dimensión y alcance del paradigma cultural latinoamericano y su contribución a la comunicación para el desarrollo, campo que en la región de América Latina se instaló por derecho propio, como resultado casi natural de nuestra particular situación de desfase histórico, económico, político y social con respecto a los restantes bloques occidentales.

"Si la primera versión latinoamericana de modernidad", plantea Barbero, "tuvo como eje la idea de Nación -llegar a ser naciones modernas-, la segunda, al iniciarse los sesenta, estará asociada a la idea de desarrollo" (Martín-Barbero, 1987, p.193). De ahí que no pocos intentos decorosos de investigación científica que se han hecho a partir de entonces en el subcontinente se dirijan "en una dirección: la superación del subdesarrollo" (Medina, 2000, p. 73). Lo anterior resulta particularmente visible en la investigación comunicológica latinoamericana, donde el abordaje de esta problemática se verifica y evoluciona a la par de cada una de las etapas o momentos por los que aquella ha venido transitando.

\section{Coordenadas metodológicas}

El artículo fue elaborado a partir de la selección, revisión y análisis de fuentes bibliográficas, provenientes en su mayoría del campo de la comunicación para el desarrollo, cuyo contenido fue examinado a la luz de los principales rasgos del paradigma cultural latinoamericano. Para ello se tuvieron en cuenta los postulados de Bourdieu, Chamboderon y Passeron, contenidos en su libro El oficio del sociólogo, quienes recomiendan, haciendo uso de un concepto acuñado por Gastón Bachelard, la vigilancia epistemológica, entendida como un ejercicio teóricamente estimulante que exige, junto a la clarificación de los conceptos, "una explicitación metódica de las problemáticas y principios de construcción del objeto que están comprendidos tanto en el material como en el nuevo tratamiento que se le aplica" (Bourdieu, Chamboderon y Passeron, 1975, p. 56).

\section{Antecedentes}

Prima entre varios autores (Sánchez Ruiz, 1985, 1986; Contreras 2000; Servaes 2000; Beltrán, 1993, 2005; Barranquero, 2005; Cimadevilla, 2011; Gumucio 2011; Aguirre 2012) un consenso bastante generalizado, prácticamente absoluto, al ubicar los inicios de este prolífico campo entre finales de la década de 1950 y la primera mitad de la siguiente década, cuando diferentes investigadores, todos norteamericanos, formularon las primeras 
teorías que vinculaban de manera directa la comunicación social con el que pasaría a ser, a la altura de 1945, el concepto clave en las ciencias sociales (Wallerstein, 2006).

Para 1986 ya había acuerdo "en que el trabajo de Daniel Lerner, The Passing of Traditional Society, publicado por primera vez en 1958, fue el principal punto de partida de la visión optimista sobre el papel de la comunicación de masas en la modernización" (Sánchez Ruiz, 1986, p. 22-23). A esta obra le siguió, en 1962, la clásica Diffusion of Innovations, de Everett Rogers, cuyo modelo, que propugnaba "la difusión generalizada de innovaciones como motor del desarrollo" (Martín-Barbero, 1987, p. 195), fue aplicado profusamente en América Latina, en el contexto del nuevo enfoque de la política norteamericana hacia la región, impulsado a partir de 1961 por la administración de J. F. Kennedy, bajo el rótulo de Alianza para el Progreso. Luego, en 1964, el trabajo de Wilbur Schramm, Mass media and national development "continuó y extendió la presuposición teórica de que los medios participaban en el desarrollo como agentes de cambio" (Sánchez Ruiz, 1986, p. 23), reafirmada, antes y después, por otros autores como Pye (1963), De Sola Pool (1963) y Frey (1966), quienes, junto a aquellos, se consideran igualmente artífices de la comunicación para el desarrollo.

"Objetivismo, empirismo estadístico, neutralidad valorativa, asepsia ideológica, énfasis metodológico" (Murgay Boils, 1979, p. 18, en Fuentes Navarro, 2005, p. 96) constituyen rasgos comunes de las teorías que configuraron el paradigma modernizador-difusionista, cuyo "valor explicativo y praxeológico" (Sánchez Ruiz, 1986, p. 8) entró en crisis mediados los sesenta, a partir de las anomalías detectadas por un grupo de investigadores latinoamericanos, precisamente desde la región en la que más tempranamente aquel dio muestras de su fracaso. Lo anterior provocó, de paso, la primera ruptura epistemológica (Bachelard, 1981) en el interior de la comunicación para el desarrollo:

[...] frente al binomio comunicación-modernización propuesto por autores como Lerner, Pye, Schramm, etc., se opone el binomio comunicación-estructura social de Beltrán, Díaz Bordenave, Marques de Melo, Ordóñez; frente al modelo psicologista de la introducción de innovaciones, se impone una constante reivindicación del contexto como punto básico para la teoría del desarrollo (Moragas 1987, 1989, en Barranquero, 2005, p. 11).

Ahora bien, las rutas conceptuales y transiciones metodológicas conducentes al nuevo paradigma distan mucho de la visión homogénea, acabada, por momentos reduccionista, desde la que se le suele representar. En tal sentido, el socorrido término de "participativo" (Servaes, 2000; Barranquero, 2005; Gumucio, 2011) quizá no sea el más preciso a la hora de captar la mixtura, el alcance y las contradicciones internas de un paradigma del cual el tipo de comunicación al que dicho término hace referencia constituye solo una parte. En todo caso, "estructural-participativo" nos parece, en ese orden, una terminología más adecuada, si tenemos en cuenta la preponderancia del primer componente en las aportaciones iniciales de la comunicología latinoamericana al desarrollo, inspirada como estuvo en la teoría de la dependencia, que, "en tanto posibilidad de interpretación crítica de las sociedades latinoamericanas, necesitaba una teoría especial de los medios de comunicación, situados en relación con ese rasgo básico del subdesarrollo" (Lenarduzzi 2001, p. 141). 
El brasileño José Marques de Melo, principal analista del período que Catalán y Sunkel han dado en llamar "momento de la corriente crítica" (1991, p. 7), se refiere así a un desplazamiento cuya primera fase tiene lugar entre la segunda mitad de los sesenta y la primera de los setenta:

$[\ldots]$ en nuestro continente emergía una vanguardia perpleja ante el impacto avasallador de la industria cultural", que tempranamente "detecta la expansión de las empresas multinacionales en América Latina y diagnóstica la diseminación de la ideología de consumo. Algunos estudios no sobrepasan la simple constatación de los nuevos fenómenos culturales. Otros dan un paso adelante, identificando los tentáculos imperialistas y señalando los peligros para la soberanía de los pueblos latinoamericanos (Marques de Melo, 1987, pp. 58-59).

Los principales focos de esta avanzada se localizan en Venezuela, Brasil, México y Chile, países que acogen, respectivamente, experiencias como el Instituto de Investigaciones de la Comunicación (ININCO), la Sociedad Brasileira de Estudos Interdisciplinares da Comunicacão (INTERCOM), el Instituto Latinoamericano de Estudios Transnacionales (ILET) y el Centro de Estudios de la Realidad Nacional (CEREN), todos lo cuales logran concentrar, indistintamente, figuras hoy consagradas del pensamiento comunicacional latinoamericano, como el belga Armad Mattelart y el brasileño Paulo Freire, los chilenos Juan Somavía y Fernando Reyes Mata, los argentinos Héctor Schmucler y Mabel Piccini, el peruano Rafael Roncagliolo, el venezolano Antonio Pasquali, además del propio Marques de Melo.

Crítico, combativo, de denuncia, fue el discurso científico construido por estos y otros investigadores, al que poco a poco irían incorporando categorías analíticas mediante las que se pretendía, más allá de obtener un acertado diagnóstico del paisaje comunicacional latinoamericano, transformar el estado de cosas existentes en él.

"La comunicación horizontal, la comunicación participativa, el derecho a la comunicación, las necesidades y recursos de la comunicación, los flujos equilibrados de la comu nicación y la tarea promotora del Estado" (Schenkel 1981, p. 1) constituyeron, además de postulados básicos del nuevo paradigma comunicacional emergente, ejes medulares del debate que en pos de un Nuevo Orden Internacional de la Información y la Comunicación (NOMIC) se libró en el transcurso de la década de 1970, con auspicio de la UNESCO y el impulso del Movimiento del Países No Alineados (MNOAL). Como basamento de este nuevo orden, se le concedió especial importancia a la formulación de políticas nacionales de comunicación (PNC), a través de las cuales se planteó directamente el enlace de esta última "con las metas globales del desarrollo" (McBride et. al, 1980, p. 169) de cada Estado nacional.

En aquel contexto, América Latina resultó "particularmente activa en el empeño de impulsar políticas comunicacionales" (Salazar et al., 2015, p. 126) en función del desarrollo integral de sus respectivos países. Tanto así, que si bien el capítulo latinoamericano sobre PNC de mediados de los setenta podría incluirse dentro de ese segundo gran momento que significó el momento corriente crítica, el cual "abarca desde finales del decenio se- 
senta hasta principios del ochenta" (Medina, 2000, p. 10), Catalán y Sunkel (1991) prefieren considerarlo una suerte de tercer momento, al constituir, según Ileana Medina, el "primer intento que se realiza en el continente por construir un paradigma autóctono" (Medina, 2000, p. 11), diametralmente opuesto a la realidad comunicacional identificada por los investigadores latinoamericanos.

De la estrecha relación que los estudiosos de entonces establecieron entre esta suerte de keynesianismo comunicacional y el desarrollo de nuestros países, dan cuenta conceptualizaciones como la de Antonio García, que concibe la

[...] política nacional de comunicación social [...] como aquella que se encuadra dentro de una estrategia de desarrollo, que orienta y regula tanto la acción del Estado como la totalidad de estructuras de participación social, que se inspira en una nueva concepción de la cultura (humanismo-social) y que hace posible la articulación y adecuación de los diversos componentes de un sistema nacional de comunicación e información (García, 1980, p 266, énfasis en el original).

Así concebidas, las PNC vendrían a dar repuesta a la Industrialización por Sustitución de Importaciones (ISI), estrategia de desarrollo sustentada en los presupuestos de estructuralismo cepalino de los años cincuenta, que se concentró "en cambiar la estructura productiva de las economías latinoamericanas y fomentar el desarrollo tecnológico" (García Rabelo, 2009, p. 68). Tal acompañamiento comunicacional, en la práctica, solo encontró una efímera concreción en los procesos vividos en Perú (1968 1975) y Chile (19870-1973), países en que los decretos "Ley del régimen del General Velasco Alvarado respecto a la socialización de la Prensa y la Radiodifusión recorrieron el continente, y la política del Presidente Salvador Allende frente a los medios de comunicación hizo noticia en la prensa mundial" (Schenkel, 1981, p. 15).

Que las buenas intenciones y los elevados propósitos de la Conferencia Intergubernamental sobre PNC de San José de Costa Rica de 1976 resultaran a la postre tan inoperantes como los de la Comisión Internacional para el Estudio de los Problemas de la Comunicación que en 1980 presentó ante la XXI Conferencia General de la Unesco el Informe MacBride se debe, entre otros factores, a que la reclamada, demandada, declamada participación se venía abajo en medio de un debate "que unificaba criterios de Estado, donde la consideración del individuo era muchas veces diametralmente opuesta", no estimándose necesario indagar sobre "el papel del hombre común, que se encuentra en el extremo de una cadena que muchas veces nacía en la sede de una agencia informativa transnacional. Sin embargo, ese eslabón final debería haber sido el origen de todas las preocupaciones" (Mattelart y Schmucler, 1983, en: Mattelart, 2006, p. 20). Como bien señala Martín-Barbero,

[...] el gran fracaso de las políticas de comunicaciones en la América Latina de los años 70 fue pensar que, poniendo solamente artículos nuevos en las leyes, iban a cambiar los procesos de comunicación, sin movilizar a los periodistas, a los artistas, investigadores, ONG, ni a las múltiples formas de organización ciudadana que existen hoy en día (2006b, p. 92). 
Pareciera entonces eufemismo hablar de participación en un escenario donde, por otra parte, la verdadera comunicación participativa, también popular y alternativa, inspirada en Paulo Freire y su concepción dialógica de la realidad, se consideraba a sí misma como una construcción solo realizable en espacios de carácter micro o sumamente localizados, cuyas prácticas no tuvieron, "salvo casos excepcionales, vinculaciones con proyectos o políticas estatales y menos aún con los foros de carácter internacional donde esas políticas se debatían" (Matta, 2011, p.12), a pesar de compartir sus postulados básicos.

Con todo, tras la estrepitosa caída del NOMIC y las PNC, "que llevará aparejada la derrota de la teoría crítica y toda pretensión reformista en la Academia y las políticas públicas regionales" (Sierra Caballero, 2009, p. 161), la comunicación participativa, popular, alternativa sería "el último refugio en el que se escondió la esperanza -y la utopía- de esa otra comunicación" (Catalán y Sunkel, 1991, p. 17), de ese otro desarrollo.

\section{El giro cultural: nuevos textos y contextos del desarrollo}

Hacia la segunda mitad de los ochenta, ya era perceptible el giro que para ese momento se producía en la investigación comunicológica latinoamericana, la cual, por primera vez en su historia, transitaba por derroteros "algo más alejados de las preocupaciones iniciales sobre el cambio social" (Barranquero, 2005, p. 14), evidenciable en la obra de figuras como Jesús Martín-Barbero, Néstor García Canclini, Guillermo Orozco y Valerio Fuenzalinda, quienes comenzaron a manejar, a partir de entonces, los hilos conductores de nuestro renovado campo comunicacional. Ileana Medina nos brinda algunas pistas para comprender los alcances de este fenómeno:

[...] en la década del ochenta los nuevos aires políticos del continente, que ya se va liberando de las dictaduras militares, propician que seamos partícipes de las grandes rupturas y desplazamientos teóricos que se han producido a nivel mundial durante la última década, y que se conoce como crisis de los paradigmas (2000, p. 12, énfasis en el original).

Es por ello que el nacimiento del paradigma cultural latinoamericano hay que verlo como parte de un proceso de mayores dimensiones: "no solo ni principalmente de deslizamientos internos al propio campo, sino de un movimiento general en las ciencias sociales" (Martín-Babero, 2006a, p. 67).

Predominarán, ahora, "enfoques más neutrales y cierta recuperación de lo empírico" (Catalán y Sunkel, 1991, p. 15), que bebe directamente del pensamiento filosófico hegemónico, al que América Latina y su investigación comunicológica se insertan desde una peculiar condición posmoderna hecha de mestizajes e hibridaciones, cuya comprensión desborda "por entero los alcances de la teoría de la dependencia o del imperialismo" (Martín-Babero, 2006a, p. 67), obligando a desplazarse desde "las influencias estructuralistas a una visión más antropológica" (Medina, 2000, p. 13), sustentada en una cierta redefinición del concepto de cultura, a partir de la cual 
[...] este ya no es concebido solamente en términos generales para referirse a todos los fenómenos de expresión de sentido - a la manera de 'todo es cultura'-, sino más bien para referirse a un campo específico y especializado en el que intervienen determinados aparatos, tecnologías, códigos, lenguajes, circuitos, etc. (Catalán y Sunkel, 1991, p. 16).

La necesidad de "reconocimiento del sujeto y la pertinencia de una teoría que parta de las percepciones de aquel" (Mattelart y Mattelart, 1991, p. 14), por una parte, y la de "situarse en los entrecruzamientos y las articulaciones de los procesos sociales, las mediaciones, para desde ahí comprender el fenómeno comunicativo" (Gámez, 2005, p. 57), por la otra, hacen que en el paradigma cultural latinoamericano converjan y se articulen, de forma orgánica, otras corrientes igualmente paradigmáticas como la recepción activa y la mediación social. Todo ello proporcionó, a no pocos investigadores que mantuvieron viva la idea de una comunicación directamente vinculada al desarrollo (Salazar et al., 2014), herramientas válidas para percibir matices y "valorar experiencias que no se pretenden totalizantes" (Hermosilla, 2007, p. 6, énfasis propio).

Esta primera y general contribución marcaría el inicio de una relación a veces tensa, por momentos distante, a la postre fecunda, entre el núcleo duro del pensamiento comunicacional latinoamericano, su línea principal, la más visible, y uno de los pocos movimientos críticos que resistió los embates de la ola neoliberal de las dos últimas décadas del siglo XX en América Latina.

Como una constante, la crítica desde la comunicación popular hacia el paradigma cultural ha presentado múltiples matices y variados tonos. He aquí un ejemplo:

Cuando hoy ciertos autores constatan, a través de sus estudios, la existencia de una cultura híbrida o constatan la existencia de los mensajes culturales, hecho que creo que es indudable, es como si detuvieran ahí su pensamiento (...) Yo creo que más allá de una descripción hay que preguntarse por el sentido que tiene esa hibridación (Matta, en Correa, 1993, p.57).

No muy distantes de estos términos en los que se refiere María Cristina Matta a Canclini se encuentran los de Gabriel Kaplún con respecto a Martín-Barbero:

[...] creo que la línea culturalista en estudios de comunicación [...] puede resultar muy ambigua [...] En el caso de Jesús Martín-Barbero [...]) creo que algunos de sus textos más famosos como De los Medios a las Mediaciones, puede tener algún uso populista muy paralizante, porque por momentos parece que lo único que hay que hacer es entender como la gente recibe, percibe y transforma lo que ve y lo que lee de los medios, pero no podemos hacer nada concreto cuando queremos trabajar con los medios (Kaplún, 2003, p. 53).

En similar dirección, Cimadevilla sostiene que, para el paradigma cultural, "importa la fuerza e impacto de los conceptos más que su posible operacionalización". Así argumenta su planteamiento:

[...] si las propuestas resultan más retóricas que vivenciales y operacionales; si todas parecen partir y compartir un mismo paradigma de construcción del 
orden; si no hay antagonismos ni contradicciones en las lecturas de los procesos, es porque los planteos se sustentan básicamente en lo discursivo. Aunque tengan serias dificultades para soportar su transferencia a escenarios de aplicación concreta (Cimadevilla, 2011, p. 105).

No lo han entendido así otros investigadores como la peruana Rosa María Alfaro, la ecuatoriana Ivonne Cevallos, los colombianos Germán Rey, José Miguel Pereira, Jorge Iván Bonilla y Julio Eduardo Benavides, el boliviano Adalid Contreras y, allende nuestros mares, Robert White, para quienes el paradigma cultural, sobre todo Martín-Barbero, abrió, en la década de los noventa, "un surco de renovación en el pensamiento académico latinoamericano sobre la comunicación popular y su nexo con el desarrollo" (Beltrán, 2005, p. 25). Lo mismo ocurrió, llegado el nuevo milenio, para la argentina Sandra Massoni y el mexicano Jesús Galindo Cáceres, principales voces en el área, respectivamente, de otras perspectivas como la comunicación estratégica y la ingeniería en comunicación, que en su variante latinoamericana han estado igualmente vinculadas al desarrollo.

La comunicación como objeto de desarrollo y no solamente condición o medio para llegar a él constituye la segunda y ya más específica contribución que debemos al paradigma cultural, revelado por Rosa María Alfaro en un libro" que "abre nuevos horizontes a la comunicación para el desarrollo y valora el aporte que pueden hacer los medios masivos" (Hermosilla, 2007, p.7). Ilustrativo de la referida contribución constituye el siguiente pasaje: "cuando la comunicación está ligada al desarrollo, lo hace no solo como aporte auxiliar y metodológico, sino como objeto mismo de transformación de la sociedad y de los sujetos que la componen" (Alfaro, 1993, p. 11, énfasis propio).

Representó, esto último, un significativo avance con respecto a la visión instrumental inherente a los paradigmas anteriores, pesado lastre heredado del difusionismo, del cual la corriente crítica, por mucho énfasis que puso en los aspectos contextuales, no logró desprenderse, atrapada como estuvo en los límites de una perspectiva que concebía los medios de comunicación, al más puro estilo althusseriano, como meros aparatos ideológicos al servicio de las clases dominantes o, cuando no, en la tradición frankfurtiana, industrias culturales tendentes "a la uniformidad y la estandarización" (Lenarduzzi, 2001, p. 143), donde casi de modo orwelliano, "apenas quedarían márgenes de opción para los individuos" (Entel, Lenarduzzi, Gerzovich, 1999, p. 6).

Algo similar es lo que sucedía, también en los setenta, con la comunicación popular, la de los espacios cotidianos, "especialmente sustentada por Paulo Freire", a la que Alfaro critica "el énfasis excesivo puesto en un proceso educativo racionalizante y centrado en el habla", que cuestionaba "mecanismos de defensa y supervivencia de los cuales la población se nutría para resistir" (Alfaro, 1993, p. 38).

Sin complejos, dispuesta a subvertir anteriores lógicas, la peruana asume la "utilidad y placer para la vida cotidiana y la relación entre sujetos", como "claves del aporte del consumidor" (Ibídem, p. 39, énfasis propio) a una comunicación para el desarrollo que, de tal modo,

Alfaro, Rosa María (1993). Una comunicación para otro desarrollo. Lima: Calandria. 
se antropologiza en grado sumo. Asunción en la que nosotros advertimos dos nuevas contribuciones del paradigma cultural, a partir de las cuales se debe reconocer y trabajar, en los proyectos de desarrollo, con "las subjetividades y los complejos modos de relacionarse con la realidad de los diversos sujetos culturales, incluyendo las complicidades y las compensaciones que cada persona requiere" (Ibídem, p. 38)².

Semejante funcionalidad la conceden Pereira, Bonilla y Benavides, en un contexto donde emergen con fuerza necesidades otras, como "el fortalecimiento de la capacidad expresiva, comunicativa y lúdica de sus habitantes" (Pereira, Bonilla y Benavides 1998, p.137), unido a lazos e intercambios que llevan a Ivonne Cevallos al convencimiento de que

[...] la comunicación en el desarrollo social contribuirá a poner en evidencia los mundos simbólicos y de sentidos de los grupos humanos y se constituirá en base para la planificación, [.. .] será un vínculo constante que facilite los consensos entre los actores sociales (Cevallos, 2001, p. 124).

Convencida Alfaro, asimismo, de la sospechosa, subversiva noción, de que los medios no constituyen ni mucho menos "máquinas infernales de serialización de lo simbólico" (Grimson, 2002, p. 76), plantea que la comunicación para el desarrollo debe trascender el ámbito comunitario y resituar "el sentido de su presencia en lo masivo, donde más bien se promuevan los diálogos sociales, la creación de consensos y disensos" (Alfaro, 1993, p. 36). Deja atrás con ello una aséptica tradición que continuaba pensando la dimensión cultural del desarrollo como algo al margen de la cultura de masas, en la que esta vendría a ser una suerte de negación y deformación.

En la misma línea de pensamiento encontramos nuevamente a Pereira, Bonilla y Benavides, para quienes, en términos de comunicación para el desarrollo, el paradigma cultural latinoamericano desbordó "los planteamientos de la corriente alternativa que estaban dedicados a identificar lo popular en tanto sinónimo -incontaminado- de todas aquellas fuerzas impugnadoras del sistema, como a oponer una barrera infranqueable entre la cultura popular y la cultura de masas" (1998, p. 134).

Se trata, ahora, de una concepción de lo popular lo suficientemente elástica para dar repuesta a una multiplicidad de redes sociales, que redimensionan el sentido de la comunicación alternativa, donde lo comunitario que la constituye, sin dejar de dar prioridad a lo local, pasa a ser entendido como "un intento también por construir esfera pública, espacios de diálogo y debate ciudadano, donde los medios pueden ser nuevas 'plazas

3 En lo referente al placer, estamos en presencia de una contribución que trasciende las fronteras del propio campo para constituir, en términos absolutos, un aporte de la comunicación a las teorías del desarrollo. Ni siquiera el desarrollo humano, concepto nacido apenas tres años antes de que Alfaro publicara su libro, reconoce el elemento lúdico como una dimensión a tener en cuenta. Haciendo uso de la tradición iluminista, el Programa de la Naciones Unidas para el Desarrollo, en cuyo Informe sobre desarrollo humano de 1990 este es definido por primera vez, se limita a ver en la adquisición de conocimientos una de las tres oportunidades esenciales para su concreción (las otras dos serían disfrutar de una vida prolongada y saludable y tener acceso a los recursos necesarios para lograr un nivel de vida decente), postura no superada en ninguno de los informes anuales emitidos a partir de entonces por el ente internacional. 
para el encuentro’" (Rey, 1997), portadoras de una agenda cuyos temas de discusión (coinciden en esto Contreras, Pereira, Bonilla y Benavides) desbordan las reivindicaciones puramente políticas, para dar cabida a cuestiones tales como el género, el racismo, la ecología, los derechos humanos, la drogadicción, la deuda externa, la salud, la educación (Pereira, Bonilla y Benavides, 1998; Contreras, 2000). Tenemos aquí otra contribución del paradigma cultural, que nos conduce directamente a la propuesta de Robert White. Desde una mirada europea, este autor ve en la construcción de una esfera pública, precisamente por carecer de ella, la principal tarea de la comunicación para el desarrollo en América Latina.

Debido a tal carencia es que White también advierte la necesidad de enfatizar, desde la comunicación, en la dimensión cultural del desarrollo, más "allá de los factores puramente económicos, técnicos y políticos", centrales en anteriores paradigmas, que fracasaron, por lo mismo, en la construcción de una esfera pública, entendida como "ese aspecto de la acción social, instituciones culturales e instancias de decisión colectiva que afectan a toda la población de una sociedad, y que articula los intereses de toda la población en el cuerpo nacional"(White, 1992, p. 2).

Como elemento novedoso, el autor introduce, en este contexto, la dramaturgia cultural, que supone "desplazar la atención hacia las iniciativas de desarrollo del pueblo y a las definiciones culturales de la situación que emergen de la confrontación y la negociación" (Ibídem, p. 17). Demasiado neutra, racional, deliberativa..., racionalmente planificada, la esfera pública moderna desconoce "las actividades en tiempo libre que operan en un nivel más imaginativo-afectivo, tales como los festivales comunales, seriales melodramáticos de ficción o el espectáculo de la política" (Ibídem).

Sin embargo, sostiene White, estos "rituales culturales públicos son por lo general un vínculo crucial entre la construcción del significado en la vida diaria y la formación de símbolos comunes en la esfera pública con los cuales la gente puede identificarse auténticamente" (Ibídem, pp. 17-18).

Para ilustrar, acude a varios ejemplos recogidos por Martín-Barbero en De los medios a las mediaciones, quien, desde un texto anterior a este, le atribuye a las fiestas una funcionalidad no muy distante de la atribuida por White a similares elementos:

Las fiestas ocupan un lugar fundamental en la cultura popular ya que $[. .$.$] proporcionan a la colectividad el espacio para descargar$ las tensiones, desahogar el capital de angustia acumulado, desviar la agresividad, activar los grupos de edad -ritos sucesivos de iniciación-y redefinir así periódicamente las relaciones de jerarquización (MartínBarbero, 1984, párr.9).

Asistimos, de tal suerte, a una redefinición del tejido comunicacional del desarrollo, portadora de una nueva tipología ${ }^{3}$ con la cual, a la altura del nuevo milenio, Contreras

4 La tipología de Contreras se encuentra precedida por otras tres, todas de Luis Ramiro Beltrán (2005, p.10): "La 'comunicación de apoyo al desarrollo' es el uso de los medios de comunicación -masivos, interpersonales o mixtos- como factor instrumental para el logro de las metas prácticas de instituciones 
resume la contribución del paradigma cultural latinoamericano durante la década recién finalizada, de una forma que no puede resultar más explícita. Bautizada como comunicación-desarrollo o comunicación con desarrollo, así se refiere a ella:

Nuevas cartografías van a derivar en otras concepciones sobre la relación entre comunicación y desarrollo. La comunicación se enriquece con los estudios de recepción y consumo cultural, diseñando un paradigma que valora las mediaciones y resignificaciones en la apropiación y uso de los mensajes desde la complejidad de las culturas. [...] Se reconceptualiza el desarrollo, para entenderse como una apuesta cultural de cambio enfocado desde los objetivos últimos del desarrollo mismo, vale decir, desde el cumplimiento de las aspiraciones de la gente. Y las concepciones sobre la comunicación abren su espacio de realización destrampándola de los límites de los medios para ubicarla en el espesor profundo de la cultura. Se hace comunicación-desarrollo desde un lugar situado, desde el lugar donde se enuncia la palabra, que es el lugar del sujeto, tomando distancia desde la revelación del 'aura' que sugiere Benjamin, o desde la apropiación crítica desafiada por Freire (Contreras, 2000, párr. 12).

Lo anterior torna más compleja la tarea, irrenunciable para este campo, de construir dispositivos orientados hacia la acción transformadora, que se justifiquen en "los sujetos historizados, con enfoques y métodos que tienen que partir de la comunicación y de las mediaciones" (Ibídem, párr. 35).

Precisamente esto es lo que han intentado hacer, en el nuevo milenio, Sandra Massoni con la comunicación estratégica y Jesús Galindo Cáceres con la ingeniería en comunicación, perspectivas entre cuyas consonancias se encuentra la preocupación de ambas por la problemática del desarrollo.

Si Massoni concibe la comunicación estratégica como un modelo de abordaje transdisciplinario "cuyo objetivo central es la idea de acción concertada para el desarrollo sostenible, entendido como cambio social conversacional" (Massoni, 2008, p. 89), para Galindo la ingeniería en comunicación vendría a constituir una suerte de punta de lanza de la comunicología que, entendida como una "visión de relaciones donde la dominación y la colaboración son procesos sociales generales que construyen a la vida social", encuentra en aquella una herramienta para promover "cursos posibles de intervención"

que ejecutan proyectos específicos en pos del desarrollo económico y social.

"La 'comunicación de desarrollo' es la creación, gracias a la influencia de los medios de comunicación masiva, de una atmósfera pública favorable al cambio que se considera indispensable para lograr la modernización de sociedades tradicionales mediante el adelanto tecnológico, el crecimiento económico y el progreso material".

A estas dos tipologías, derivadas de las teorías norteamericanas que vimos en las primeras páginas del artículo, le sucede una tercera con la cual Beltrán define la experiencia latinoamericana de los setenta en materia de comunicación para el desarrollo. Así pues, "La comunicación alternativa para el desarrollo democrático es la expansión y el equilibro en el acceso de la gente al proceso de comunicación y en su participación en el mismo empleando los medios - masivos, interpersonales y mixtos -para asegurar, además del avance tecnológico y del bienestar material, la justicia social, la libertad para todos y el gobierno de la mayoría" (Ibídem, p. 21). 
dirigidos a "aliviar males emergentes" y al logro de "un desarrollo sustentable" (Galindo Cáceres, 2012b, p. 7).

En medio de la mixtura ${ }^{4}$ que configura la propuesta de la argentina, las mediaciones ocupan, al igual que en Barbero, un lugar central. Pero a diferencia de este, ella prefiere pensarlas en acción. Así, pues, las concibe como "dispositivos articuladores [.. . ] de actores, prácticas e imaginarios" (Massoni y Mascotti, 2000, p. 1), en un mundo fluido donde "no hay un narrador centrado, ni emisores tan definidos como fuentes, ni contenidos tan unívocos, sino transformaciones permanentes en las que todos son actores múltiples" (Massoni, 2008, p. 90).

Otra de las categorías centrales en el modelo de Massoni constituye las matrices socioculturales, esto es: lógicas de funcionamiento desde las cuales cada actor (personas, grupos e instituciones clave, etc.) establece la comunicación con el concurso de otros tres componentes, a saber: los modos (formas de comunicación, códigos, redes de interacción, etc.), los espacios (lugares de intercambio, circulación y resemantización de las novedades, etc.) y los saberes (visión del problema, conceptualizaciones, destrezas, etc.) (Massoni, 2007). Aquí, al igual que en las mediaciones, reconoce el aporte de los estudios culturales, especialmente los estudios críticos de las audiencias de Orozco y Canclini, a la conformación de una categoría que permite introducir, cual vimos en Alfaro, "la problemática del placer en el marco de las determinaciones socioculturales: si alguien hace algo es porque hay alguna ganancia de algún tipo" (Massoni, 2000, p. 100).

Bajo tales premisas el desarrollo no pude ser definido de otra manera que como transformación, "como un cambio siempre desde dentro aun cuando no estemos muy acostumbrados a pensar así a la innovación", donde el aporte de la comunicación no estaría más "en venderle soluciones a la gente", sino "en ofrecerles la posibilidad de encontrarlas en conjunto" (Massoni, 2008, p. 93).

Vemos en la densidad operativa que le impregna el paradigma cultural a la comunicación para el desarrollo por medio de la perspectiva estratégica de Massoni, una contribución cuyos rasgos se encuentran igualmente presentes en Galindo Cáceres.

En su crítica a la orientación marcadamente político-economicista que históricamente han acusado los modelos de desarrollo, este autor advierte una cuestión medular: "la cultura como cosmología y matriz creativa de lo social" (Galindo, 2012b, p. 16) ha estado siempre relegada a un plano secundario. Sin embargo, sostiene Galindo, "la cultura es una visión que integra lo que la política y la economía no han logrado ni intentado" (Galindo, 2012b, p.21), esto es, la fuerza subjetiva de lo simbólico. Por tal motivo, al igual que White, Galindo asume "la cultura como una figura de organización social del mundo público", desde una perspectiva que se declara antropológica y sobre todo comunicológica, "donde

\footnotetext{
$5 \quad$ Otras de sus fuentes son los neoparadigmáticos chilenos Humberto Matura y Francisco Varela, el belga de origen ruso Ilya Prigogine y el siempre recurrido Edgar Morin. Se nutre, asimismo, del pensamiento ambiental latinoamericano, específicamente de autores como Enrique Leff, Augusto Maya, Carlos Galano y Felipe Ángel.
} 
la promoción y la gestión cultural son un posible corazón de la ingeniería social" (Galindo, 2012b, p. 11), la cual constituye el núcleo duro de su propuesta.

Sin hacer mención explícita a nombres o figuras, es del paradigma cultural que Galindo recupera para su ingeniería en comunicación la noción de lo público, que hace converger con la de lo privado proveniente de la comunicación organizacional, ahora como "parte de un solo programa, un gran espacio conceptual-metodológico de la comunicación para la acción constructiva" (Galindo, 2012a, p.6). Su propuesta deriva en la posibilidad de intervenir comunicacionalmente en casi cualquier ámbito o esfera de lo social:

En un sentido macro-social, la propaganda y la publicidad, el proselitismo político y el religioso, la mercadotecnia, la economía de mercado, la industria del entretenimiento, la industria cultural, la ingeniería industrial, el trabajo con redes sociales, las técnicas de desarrollo de comunidad, el trabajo social. Y en un sentido micro social, las terapias, las técnicas en formación de grupos, la propuesta del desarrollo humano, el análisis institucional, el socioanálisis, la investigación acción-participativa, la praxiología, la socio-praxis, la comunicación en organizaciones (Galindo, 2011, pp. 36-37).

Ya sea como "momento relacionante de la diversidad sociocultural y, por lo tanto, espacio del cambio, de la transformación" (Massoni, 2008, p. 91), o "como matriz de la organización y el desarrollo social" (Galindo, 2012a, p. 11), la comunicación, tanto en Massoni como en Galindo, se encuentra llamada a jugar un papel determinante en los procesos del desarrollo latinoamericano, donde la figura del comunicador, ahora estratégico, es la de un profesional "con capacidad de interpelar la dinámica social para operar crítica y valorativamente en su dimensión comunicativa" (Massoni y Mascotti, 2000, p. 7). Su tarea, además de atender emergencias, proponer opciones de desarrollo y mantener la salud del cuerpo social (Galindo, 2012a), implica reconocer la diversidad sociocultural, reconocimiento con el cual, a diferencia de los estudios culturales, ya podemos hacer algo: "indagar los posibles puntos de articulación de las diferencias en función de intereses y necesidades de grupos sociales que se reconocen como bien distintos, para operar desde allí con relación a un objetivo" (Massoni y Mascotti, 2000, p. 3).

$\mathrm{Si}$, como todo indica, la comunicación estratégica y la ingeniería en comunicación constituyen perspectivas que desde su equivalencia han hecho posible una refundación del pensamiento comunicacional latinoamericano en lo que va del siglo XXI, puede que, finalmente, nos encontremos en condiciones de materializar, en nuestro campo, aquella promesa de la que nos hablaba Charles Wright Mills (1969) en su Imaginación sociológica5. Cuando menos, el camino y la ruta a seguir están planteadas.

5 En este libro Mills recoge el siguiente pasaje de Paul Lazarsfeld: "la sociología no aún está en la fase que pueda proporcionar una base segura para la ingeniería social... Tardaron las ciencias naturales unos 250 años, desde Galileo hasta comienzos de la revolución industrial, en adquirir influencia importante en la historia del mundo. La investigación social empírica tiene su historia de tres a cuatro décadas. Si esperamos de ella soluciones rápidas a los mayores problemas del mundo, si no le exigimos más que resultados inmediatamente prácticos, no haremos otra cosa que perturbar su curso natural" (Lazarsfeld, 1948, en: Wright Mills, 1969, p. 116) 


\section{Conclusiones}

¿En qué medida el cambio de paradigma que se produce en el contexto de la investigación latinoamericana durante los 80, propició la ocurrencia de similar proceso en el interior de la comunicación para el desarrollo a partir de la década inmediatamente posterior?

Si por paradigma entendemos, con Thomas S. Khun, las "realizaciones científicas universalmente reconocidas que, durante cierto tiempo, proporcionan modelos de problemas y soluciones a una comunidad científica" (Kuhn, 1971, p. 13), podemos afirmar, a ciencia cierta, que la tipología de la que da cuenta Contreras (2000) para caracterizar los nuevos derroteros de las relaciones entre comunicación y desarrollo puede ser elevada al rango de paradigma, al igual que el universalmente reconocido paradigma cultural latinoamericano.

Por una parte, los representantes de la llamada comunicación de desarrollo (Contreras, 2000), con base en los presupuestos de la comunicación popular, han sabido señalar certeramente las anomalías de la ciencia normal que se venía haciendo en esta. Y por la otra, han identificado problemáticas y demostrado ellos mismos soluciones plausibles mediante las herramientas conceptuales Incautadas ¿SERÁ INCAUTADAS? del paradigma cultural, el cual, de paso, propició el nacimiento de otro, cuyo eje articulador, el trinomio comunicación-cultura-desarrollo, comenzó a ser interpelado a partir de los noventa y todavía hoy continúa ofreciendo respuestas plausibles para no pocos comunicólogos, desde perspectivas como la comunicación estratégica y la ingeniería en comunicación social.

Sin embargo, las aportaciones del referido paradigma a la comunicación para el desarrollo no siempre han contado con el debido reconocimiento por parte de sistematizadores y hagiógrafos, incluso los más preclaros, quienes, o bien terminan desdibujándolas en la inmensa vastedad ¿SERÁ VASTEDAD? del paradigma participativo (Beltrán, 2005; Barranquero, 2005), o simplemente terminan desconociéndolas, de una manera poco menos que sospechosa (Servaes, 2000; Gumucio, 2011; Aguirre, 2012), en la que se advierte esa lucha por el monopolio de la autoridad científica del campo referida por Bourdieu (Bourdieu, 1990).

Sirva este trabajo, cuando menos, como un acto de reivindicación científica.

\section{Referencias bibliográficas}

Alfaro, R. M. (1993). Una comunicación para otro desarrollo. Lima, Perú: Calandria.

Aguirre Alvis, J. L. (2012). Enfoques teóricos para una comunicación orientada al desarrollo y retos actuales para una comunicación y desarrollo desde la diversidad. Punto Cero, 17(24), 58-66.

Bachelard, G. (1981). La formación del espíritu científico. México D. F., México: Siglo XXI.

Barranquero, A. (2005). Latinoamérica en la ruptura del paradigma de la comunicación para el desarrollo. El recorrido de los pioneros en la búsqueda de alternativas democráticas. Punto Cero 10(11), 7-22.

Beltrán, Luis. R. (1993). Comunicación para el desarrollo en Latinoamérica. Una evaluación sucinta al cabo de cuarenta años. Recuperado: http//: www.comminit.com/es/node/150404. 
(2005). La comunicación para el desarrollo en Latinoamérica: un recuento de medio siglo, Recuperado de http: www.portalcomunicacion.com/both/temas/lramiro.pdf.

Bourdieu, P. (1990). Sociología y cultura. México D. F., México: Editorial Grijalbo.

Bourdieu, P.; Chamboderon, J. C. E Passeron, J. C. (2002). El oficio del sociólogo Presupuestos epistemológicos. Buenos Aires, Argentina: Siglo XXI Editores.

Catalán, C. E Sunkel, G. (1991). La tematización de las comunicaciones en América Latina En Comunicación, (76), 4-26,

Cevallos, I. (2001). "Los espacios de la comunicación en el desarrollo social". En I. Rodrigo Mendizábal E L.Cucurella, (editores) Comunicación en el tercer milenio. Nuevos escenarios y tendencias (pp.121-132). Quito, Ecuador: Ediciones Abya-Yala.

Cimadevilla, G. (2011). Cinco tesis y una semblanza. Trayectos académicos en la convergencia comunicación-desarrollo. Revista ALAIC, (8-9), 100-110,

Contreras, A. (mayo-julio 2000). Comunicación-desarrollo para otro occidente. Razón y palabra, (18). Disponible en http://www.razonypalabra.org.mx/N/N86/N86/05_SalazarSalazarNaranjo_V86.pdf.

Correa, C. J. (1993). "María Cristina Mata: el consumo desde una perspectiva crítica (entrevista)". Comunicación, (81), pp. 56-59

De Sola Pool, I. (1963). "The Mass Media and Politics in the Modernization Process" En L. W. Pye (Eds.), Communications and Political Development (234-253). Nueva Jersey, USA: Princeton University Press.

Entel, A.; Lenarduzzi, V. E Gerzovich, D. (1999). "La escuela de Frankfurt en América Latina". En Escuela de Frankfurt. Razón, arte y libertad. Buenos Aires, Argentina: Ed. Eudeba.

Frey, W. (1966). "The Mass Media and Rural Development in Turkey". Rural Development Research Report, (3.).

Fuentes Navarro, R. (2005). Everett M. Rogers (1931-2004) y la investigación Latinoamericana de la comunicación. Comunicación y Sociedad, (4), 93-125.

Galindo Cáceres, J. (2009). Comunicología, etnometodología y comunicometodología. La comunicación como acción y como representación reflexiva constructiva. Razón y Palabra (67). Disponible en www. razonypalabra.org.mx/N/n67/actual/1 jgalindo.html

(2011). Metodología en ingeniería en comunicación social. Apuntes generales sobre su programa metodológico: el diagnóstico, el diseño de la intervención social, y la aplicación técnica de la intervención. Disponible en http://ieie.udistrital.edu.co/pdf/TEXTO\%202\%20 Metodolog\%C3\%ADa\%20en\%20\%20Ingenier\%C3\%ADa\%20en\%20Comunicaci\%C3\%B3n\%20Social. pdf....

(2012a). Comunicología, Ingeniería en Comunicación Social y Comunicación Estratégica. Construyendo un programa de Altos Estudios en comunicación. Disponible en http://www. tendencias21.net/estrategar/docs/jesus_galindo_comunicologia,_ingenieria_en_comunicacion_ social_y_comunicacion_estrategica.pdf.

(agosto-octubre 2012b). Ingeniería en comunicación social, comunicología y desarrollo. Elementos para una crítica de la promoción y la gestión cultural en México. Razón y Palabra, 80. Disponible en www.razonypalabra.org.mx/N/N80/M80/02_Galindo_M80.pdf

Gámez, N. (2005). Los usos del concepto de mediación en el campo de la comunicación: crítica y perspectivas. (Tesis de Maestría inédita). Universidad de La Habana, Cuba. 
García, A. (1980). ¿Comunicación para la dependencia o para el desarrollo? Quito, Ecuador: Ciespal.

García Rabelo, M. (2006). La interconexión de factores endógenos y exógenos en el subdesarrollo y el desarrollo. El estructuralismo y la dependenciaINCOMPLETO

García Rabelo, (coord.) Las teorías acerca del subdesarrollo y el desarrollo: una visión crítica (pp.65-79). La Habana, Cuba: Félix Varela.

Grimson, A. (2002). Las industrias culturales en la integración latinoamericana. Punto Cero, 7 (5), 76-78.

Gumucio-Dragón, A. (2011). Comunicación para el cambio social: clave del desarrollo participativo. Signo y Pensamiento, 30 (58), 26-39.

Hermosilla, M. E. (2007). Comunicación para el Desarrollo en América Latina: ¿Tiene aún sentido? Comunicación: estudios venezolanos de comunicación, (137), 4-8.

Kaplún, G. (2003). Comunicación participativa y Educación Popular en el contexto barrial y de las organizaciones. En Ayme Plasencia Pons (Eds.): Enfoques sobre la comunicación en la gestión de proyectos comunitarios (pp.53-70). La Habana, Cuba: Ediciones Unión.

Kuhn, T. S. (1971) La estructura de las revoluciones científicas. México D. F., México: Fondo de Cultura Económica.

Lenarduzzi, V. (2001). La prohibición de la imaginación: recepción y usos de la escuela de Frankfurt en América Latina. En M. I. Vassallo de Lopes, Immacolata y R. Fuentes Navarro (comp.), Comunicación objeto y estudio. Perspectivas reflexivas latinoamericanas (pp. 127-148). Guadalajara, México: ITESO.

McBride, S. (1980). Un mundo, voces múltiples. Comunicación e información en nuestro tiempo. México D. F.: Fondo de Cultura Económica.

Marques de Melo, José (1984), "Teoría e investigación de la comunicación en América Latina. Balance preliminar de los últimos 25 años". Estudio sobre las Culturas Contemporáneas, 1 (2), 53-72.

Martín-Barbero, J. (1984). Cultura popular comunicación de masas. Disponible en http://www2.uned. es/ntedu/asignatu/3JMartinBarbero.htm.

(1987). De los medios a las mediaciones. Barcelona, España: Gustavo Gili.

(2006a). Aventuras de un cartógrafo mestizo en el campo de la comunicación. En Hilda Saladrigas (comp), Comunicología. Temas Actuales (pp.58-70). La Habana, Cuba: Félix Varela.

(2006b). La integración como agenda mediática. En Blanca Rosales (Coord.), Comunicación para la integración. Prácticas y desafíos de la región andina (pp. 87-94). Lima, Perú: Comunidad Andina.

Massoni, Sandra \& Mascotti, Mariana (2000). Apuntes para la comunicación en un mundo fluido: Mediación no es mediar. Disponible en http://www.eca.usp.br/associa/alaic/chile2000/1 GT\%20 2000Com\%20Tecnologia\%20e\%20dessarrollo/SandraMassoni.doc.

Massoni, S. (2000), Estrategias de comunicación: tiempos de investigarnos vivos. Comunicación y Sociedad, (37), 87-109

(2007). Estrategias. Los desafíos de la comunicación en un mundo fluido. Buenos Aires, Argentina: Homo Sapiens Ediciones Rosario.

(2008). Comunicación y desarrollo. Encuentros en la diversidad. En: R. Thornton, \& G. Cimadevilla, (editores), Grises de la extensión, la Comunicación y el Desarrollo (pp.87-100). Buenos Aires, Argentina: Inst. Nacional de Tecnología Agropecuaria -INTA-. 
Matta, M. C. (2011). Comunicación popular: continuidades, transformaciones y desafíos. Oficios terrestres, (26), pp. 1-22.

Mattelart, A. \& Mattelart, M. (1991). La recepción: el retorno al sujeto. Diálogos de la Comunicación, (30), $10-17$.

Mattelart, A. (2006). Entre el Nuevo Orden Mundial de la Información y la Comunicación y la Cumbre Mundial sobre la Sociedad de la Información. Telos, (76), 13-26

Medina, I. (2000). Desde el otro lado. La Habana, Cuba: Pablo de la Torriente Brau.

Pereira, J.M; Bonilla, J.I. E Benavides, J. E. (1998). La comunicación en contextos de desarrollo: balances y perspectivas. Signo y Pensamiento, 17 (32), 119-138.

PNUD (1990). Informe sobre Desarrollo Humano. Nueva York, USA: ONU. Disponible en: hdr.undp.org/sites/ default/files/hdr_1990_es_completo_nostats.pdf

Pye, L. W. (1963). Communications and Political Development. Nueva Jersey, USA: Princet University Press.

Rey, G. (1997). Otras plazas para el encuentro. En R. M. Alfaro (Eds.), Escenografías para el diálogo (pp. 19-48). Lima, Perú: Calandria.

Rogers, E. M. (1962). Diffusion of innovations. New York, USA: The Free Press.

Salazar Martínez, R. A.; Salazar Díez, R.A. E Naranjo Almarales R. M. (abil-junio 2014). "Relaciones entre comunicación y desarrollo local. Diatriba contra reduccionismo y coordenadas para su articulación". Razón y palabra, (86). Disponible en http://www.razonypalabra.org.mx/N/N86/N86/05 SalazarSalazarNaranjo_V86.pdf.

Salazar Martínez, R.A. (2015). "Políticas nacionales de comunicación en el nuevo modelo cubano". Temas (81-82), 125-132.

Sánchez Ruiz, E. (1985). "Notas sobre el problema de la validación empírica en la sociología del desarrollo". Revista Encuentro, 2 (2), 7-25.

(1986). Réquiem por la modernización: perspectivas cambiantes en estudios del desarrollo. Cuadernos de Difusión Científica (7), Serie Comunicación y Sociedad.

Schenkel, P. (1981). Políticas Nacionales de Comunicación (prólogo). Quito, Ecuador: Ed. Época.

Schramm, W. (1964). Mass Media and National Development. California, USA: Stanford University.

Servaes, J. (2000). Comunicación para el desarrollo: tres paradigmas, dos modelos". Temas y problemas de la comunicación, 10, 5-28.

Sierra Caballero, F. (2009). Economía política de la comunicación y teoría crítica. Apuntes y tendencias. Revista Científica de Información y Comunicación, (6) 149-171.

Wallerstein, I. (2006). Abrir las Ciencias Sociales. Madrid, España: Siglo XXI.

White, R. (1992). Análisis cultural en la comunicación para el desarrollo: el rol de la dramaturgia cultural en la creación de una esfera pública. Diálogos de la Comunicación, 34, 1-21.

Wright Mills, C. (1969). La imaginación sociológica. La Habana, Cuba: Instituto del Libro.

Para una periodización de la investigación comunicológica latinoamericana, consúltense a Catalán y Sunkel (1991). 